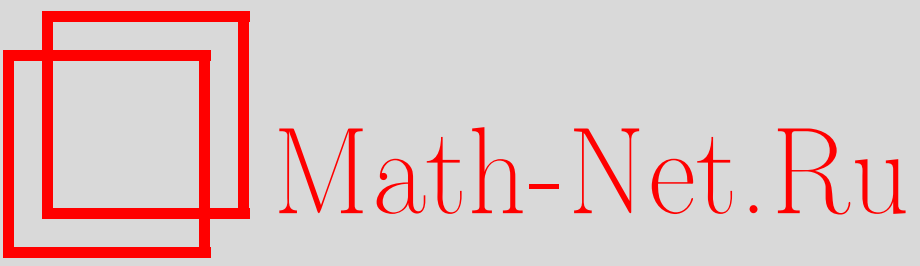

Э. Ю. Лернер, Фейнмановские интегралы от р-адического аргумента в импульсном пространстве III. Перенормировка, ТМФ, 1996, том 106, номер 2, 233-249

DOI: https://doi.org/10.4213/tmf1110

Использование Общероссийского математического портала Math-Net.Ru подразумевает, что вы прочитали и согласны с пользовательским соглашением

http://www . mathnet.ru/rus/agreement

Параметры загрузки:

IP : 3.82 .47 .9

26 апреля 2023 г., 07:03:40 


\title{
ФЕЙНМАНОВСКИЕ ИНТЕГРАЛЫ ОТ $p$-АДИЧЕСКОГО АРГУМЕНТА В ИМПУЛЬСНОМ ПРОСТРАНСТВЕ III. ПЕРЕНОРМИРОВКА
}

\begin{abstract}
Найдены инфракрасная и ультраффиолетовая серии полюсов безмассовых фейнмановских интегралов от $p$-адического аргумента с произвольными степенями пропагаторов. В рамках схемы минимальных вычитаний для аналитической $R^{*}$-операции доказана конечность перенормированных амплитуд. Даны простые формулы для вычисления контрчленов (вершинных частей) в координатном и импульсном пространствах.
\end{abstract}

\section{1. ВВЕДЕНИЕ}

Один из аргументов в пользу изучения $p$-адических моделей состоит в том, что $p$ адические теории $[1,2]$ могут оказаться полезными с методологической точки зрения в сложных задачах квантовой теории поля и статистической механики, поскольку ультраметричность $p$-адической нормы сильно облегчает вычисления. В частности, если в обычной вещественной квантовой теории поля исследование различных схем перенормировки проводится в рамках $\alpha$-представления [3, 4], то в $p$-адическом случае основой доказательства теоремы о перенормируемости [5] могут служить явные выражения для фейнмановских амплитуд [6, 7].

Данная статья посвящена исследованию $p$-адического аналога аналитической перенормировки, введенной Спиром, и аналитической $R^{*}$-операции, введенной в вещественном случае в работах Четыркина, Ткачева, Смирнова (см. монографию [8] и ссылки в ней). Теорема о конечности аналитически перенормированных фейнмановских интегралов от $p$-адического аргумента (ФИ $p$-А) для $\varphi_{d}^{4}$-модели в рамках теории обобщенных функций была доказана в [5]. Возможность применения других перенормировочных схем на простых примерах была продемонстрирована в [9]. $(4-\varepsilon)$-разложение и размерная перенормировка амплитуд $\varphi_{d}^{4}$-теории рассматривались в [10]. В работе [11] оператор перенормировки в $p$-адической $(\bar{\psi} \psi)^{2}$-модели описан как нормализующее отображение к преобразованию ренормгруппы Каданова. Это преобразование задает конечномерную динамическую систему в пространстве констант связи. В этой статье мы выпишем серии полюсов безмассовой $p$-адической амплитуды с однородными пропагаторами и с пропагаторами, обрезанными в нуле или на бесконечности (аналогичные задачи в вешественном случае были решены в работах [12-15]). В рамках схемы мини- 
мальных вычитаний мы докажем конечность аналитически $R^{*}$-нормированных $p$-адических амплитуд с однородными пропагаторами.

Основой наших доказательств является полученное в $[16,17]$ представление $Ф И$ с однородным пропагатором в виде линейной комбинации $Ф И$ с ограниченным пропагатором. Коэффищиенты этой линейной комбинации представляют собой вакуумные амплитуды с пропагатором, обрезанным в нуле или на бесконечности. Заметим, что это представление опирается на простое следствие ультраметричности $p$-адической нормы, заключающееся в том, что значение $Ф И$-А с пропагатором, являющимся функцией от $\left\|k_{l}\right\|\left(1-\chi r\left(k_{l}\right)\right)$, не зависит от конкретных значений внешних импульсов, принадлежаших шару $\Omega_{r}=\left\{x \in Q_{p}^{d}:\|x\| \leq r\right\}$. Здесь $k_{l} \in Q_{p}^{d}$ - импульс внутреннего ребра $l$, а $\chi_{r}$ - индикатор шара $\Omega_{r}$.

В этой работе, следуя [18], мы будем называть остовным подграфом связного графа $G$ любой его подграф $H$, множество вершин которого $V(H)$ совпадает с $V(G)$ (в статье [17] мы дополнительно предполагали связность остовного подграфа $H$ ). Очевидно, что сушествует взаимно однозначное соответствие между остовными подграфами $H$ графа $G$ и наборами непересекаюшихся связных подграфов $\pi \equiv \pi(H)=\left\{H_{1}, \ldots, H_{n}\right\}$ таких, что $\bigcup_{i} V\left(H_{i}\right)=V(G)$ (подграфы из набора $\pi(H)$ суть компоненты связности графа $H$ ). Мы будем употреблять одни и те же обозначения как для некоторых совокупностей остовных подграфов $H$, так и для множеств соответствуюших им наборов $\pi(H)$. Сушественным подграфом [15] называется подграф,, содержаший все внешние вершины графа $G$ в одной компоненте связности. Пусть $\mathcal{P}(G)$ - совокупность всех остовных подграфов графа $G ; \mathcal{P}^{\prime}(G)$ - часть $\mathcal{P}(G)$, состоящая из сушественных подграфов. Если $H \in \mathcal{P}^{\prime}(G)$, то множество внешних линий графа $H$ мы определим как множество $E$ всех внешних линий исходного графа $G$. Заметим, что это нестандартное определение; обычно в качестве внешних линий графа $H$ рассматривают более богатое множество. Звездой $S t(v)$ вершины $v$ называется множество всех ребер, инцидентных вершине $v$. Определения всех остальных терминов теории $Ф И$, используемых в данной работе, можно найти в [8] (см. также приложение к [13]).

Обозначим через $F_{G}(q ; \lambda ; m, M) \Phi И ~ p-\mathrm{A}$ :

$$
\left.\int d \underline{p} \prod_{l \in L(G)}\left\|k_{l}\right\|^{\lambda_{l}}\left(\chi_{M}\left(k_{l}\right)-\chi_{m}\left(k_{l}\right)\right)\right|_{k=k(q, \underline{p})} .
$$

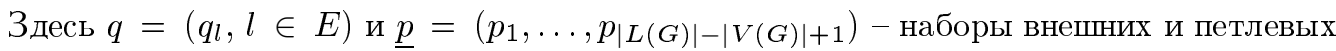
импульсов, соответственно, $\lambda_{l}, l \in L(G)$, - комплексные параметры (по поводу остальных обозначений см. $[16,17])$. Основной объект наших исследований - интеграл $F_{G}(q ; \lambda) \equiv F_{G}(q ; \lambda ; 0, \infty)$. Простейший вариант упомянутого выше представления имеет вид

$$
F_{G}(q ; \lambda)=\sum_{H_{1} \in \mathcal{P}^{\prime}(G)} \sum_{H_{2} \in \mathcal{P}\left(H_{1}\right)} g_{G / H_{1}}(\lambda ; 0, m) F_{H_{1} / H_{2}}(q ; \lambda ; m, M) g_{H_{2}}(\lambda ; M, \infty) .
$$

Здесь $g_{H}(\cdot)$ - вакуумньй интеграл $F_{H}(0 ; \cdot) ; \quad M \geq \max _{l \in E}\left\|q_{l}\right\|$ и $m<\min _{E^{\prime} \subset E}\left\|\sum_{l \in E^{\prime}} q_{l}\right\|$. Мы полагаем, что интегралы, индексируемые графами, не имеющими ребер, равны единице по определению. В формуле (1) и везде ниже будем предполагать, что вектор внешних импульсов $q$ не принадлежит сингулярному множеству Симанзика $\bigcup_{E^{\prime} \subset E}\left\{q \in Q_{p}^{d|E|}: \sum_{l \in E^{\prime}} q_{l}=0\right\}$. Несколько более сложные формулы 
возникают, если в (1) ограничиться суммированием только по множеству $P(G)$ остовных подграффов графа $G$, все компоненты связности которых являются полными подграфами. А именно, имеет место представление, найденное в работе [17],

$$
F_{G}(q ; \lambda)=\sum_{H_{1} \in P^{\prime}(G)} \sum_{H_{2} \in P\left(H_{1}\right)} f_{G / H_{1}}(\lambda ; 0, m) \Phi_{H_{1} / H_{2}}(q ; \lambda ; m, M) f_{H_{2}}(\lambda ; M, \infty) .
$$

Здесь $\Phi_{G}(q ; \lambda ; m, M)$ есть сумма $Ф И$

$$
\left.\sum_{S \in \tau(G)} \int d \underline{p} \prod_{l \in L(S)}\left(\chi_{M}\left(k_{l}\right)-\chi_{m}\left(k_{l}\right)\right)\left\|k_{l}\right\|^{\lambda_{l}} \prod_{l \in L(G) \backslash L(S)} \chi_{m}\left(k_{l}\right)\left\|k_{l}\right\|^{\lambda_{l}}\right|_{k=k(q, \underline{p})},
$$

где $\tau(G)$ - набор связных остовных подграфов граффа $G ; f_{G}(\cdot)$ есть вакуумный интеграл $\Phi_{G}(0 ; \cdot) ; P^{\prime}(G)$ - часть $P(G)$, состояшая из сушественных подграфов. Очевидно, что $\Phi_{G}(q ; \lambda ; 0, m)=F_{G}(q ; \lambda ; 0, m)$.

Заметим, что первое суммирование в формулах $(1),(2)$ достаточно вести по множеству $\widetilde{\mathcal{L}}(G)$ подграфов $H$ таких, что граф $G / H$ одночастично неприводим. Второе суммирование в этих формулах достаточно вести по множеству $\widehat{\mathcal{L}}(G)$ подграфов $H$, каждая компонента которых представляет собой одночастично неприводимый подграф. В дальнейшем мы будем использовать обозначение $\mathcal{L}(G)$ для пересечения множеств $\widetilde{\mathcal{L}}(G)$ и $\widehat{\mathcal{L}}(G)$.

Разложения (1) и (2) позволяют свести анализ сингулярностей интеграла $F_{G}(q ; \lambda)$ к анализу полюсов $\lambda^{*}=\left(\lambda_{l}^{*}, l \in L(G)\right)$ вакуумных интегралов с пропагатором, обрезанным в нуле или на бесконечности. Более того, $R^{*}$-операция сводится к устранению расходимостей из вакуумных интегралов. Явные формулы для этих интегралов (см. раздел 2 работы [17]) приводят к существенному упрошению доказательств результатов теории $R^{*}$-операции в $p$-адическом случае.

Статья организована следуюшим образом. В разделе 2 мы выпишем ультрафиолетовую (УФ) и инфракрасную (ИК) серии полюсов $Ф И F_{G}(q ; \lambda ; m, \infty), F_{G}(q ; \lambda ; 0, M)$ и $F_{G}(q ; \lambda)$. Из этих результатов следует полное отсутствие ИК-полюсов у интеграла $F_{G}(q ; \lambda)$, если степени пропагаторов удовлетворяют соотношениям $\lambda_{l}>-d$ для всех $l \in L(G)$ и $\sum_{l \in S t(v)}\left(\lambda_{l}+d\right)=2 d$ для всех внутренних вершин $v$ (таким соотношениям удовлетворяют, например, степени пропагаторов в диаграммах $\varphi_{4}^{4}$ и $\varphi_{6}^{3}$-моделей). В разделе 3 мы определим аналитическую $R^{*}$-операцию и докажем конечность $R$-нормированных амплитуд $F_{G}(q ; \lambda ; m, \infty), \widetilde{R}$-нормированных амплитуд $F_{G}(q ; \lambda ; 0, M)$ и $R^{*}$ нормированных амплитуд $F_{G}(q ; \lambda)$. Последний результат обобшает теорему о конечности перенормированных амплитуд безмассовой $\varphi^{4}$-теории, доказанную в [5]. В разделе 4 мы определим $R^{*}$-операцию для координатного представления $\Phi И ~ p$-А и установим связь между вершинными частями, возникающими при устранении расходимостей в координатном и импульсном пространствах. Эта связь дает возможность находить контрчлены в импульсном пространстве с помошью простых “координатных" формул.

\section{2. ПОЛЮСЫ ФЕЙНМАНОВСКИХ ИНТЕГРАЛОВ В ИМПУЛЬСНОМ ПРОСТРАНСТВЕ}

Как показано в $[16,17]$, аналитическое продолжение мероморфной функции $F_{G}(q, \lambda)$ определено для любых значений параметров $\lambda$, если граф $G$ имеет не менее двух внеш- 
них вершин и не содержит отделимых подграфов. Если последние условия не выполнены и граф $G$ нетривиален, то положим, что $F_{G}(q, \lambda) \equiv 0$ по определению. Заметим, что интеграл $f_{G}(\lambda ; M, \infty)$ имеет непустую область сходимости только, если граф $G$ не содержит лепестков. В противном случае мы также положим, что $f_{G}(\lambda ; M, \infty) \equiv 0$. Все остальные интегралы в импульсном пространстве, рассматриваемые в данной статье, определены как мероморфные функции параметров $\lambda$ для любых графов $G$.

Обозначим через $\mathcal{Q}$ (через $\mathcal{Q}^{\prime}$ ) совокупность двусвязных редуцированных графов $Q=G / H$ таких, что $H$ принадлежит $P(G)$ (принадлежит $P^{\prime}(G)$ ). Пусть $\mathcal{Q}_{0}^{\prime}-$ совокупность всевозможных редуцированных графов $Q=G / H$ таких, что $H \in P^{\prime}(G)$ и $\left|V\left(H^{\prime}\right)\right|=1$ для всех компонент связности $H^{\prime}$ графа $H$, кроме компоненты с множеством вершин $V^{\prime} \supseteq V_{\text {ext }}$. Через $\mathcal{H}$ (через $\mathcal{H}_{0}$ ) обозначим множество всевозможных (множество полных) двусвязных подграфов графа $G$. Пусть $\beta(G)=|L(G)|-|V(G)|+1$, $\operatorname{Ind}(G)=d \beta(G)+\sum_{l \in L(G)} \lambda_{l}$.

ТЕОрема 1. Пусть $G$ - связный граф, не содержащий отделимых подграфов, и $|E(G)| \geq 2$. Тогда

$$
F_{G}(q ; \lambda) \prod_{l \in L(G)}\left(1-p^{\lambda_{l}+d}\right) \prod_{H \in \mathcal{H}_{0}}\left(1-p^{\operatorname{Ind}(H)}\right) \prod_{Q \in \mathcal{Q}_{0}^{\prime}}\left(1-p^{\operatorname{Ind}(Q)}\right)
$$

- челая функиия от переменных $\lambda$.

Заметим, что произведение

$$
\Phi_{G}(q ; \lambda ; m, M) \prod_{l \in L(G)}\left(1-p^{\lambda_{l}+d}\right), \quad 0<m, M<\infty
$$

представляет собой целую функцию от переменных $\lambda$, поскольку

$$
\Phi_{G}(q ; \lambda ; m, M)=\sum_{S \in \tau(G)} F_{S}(q ; \lambda ; m, M) \prod_{l \in L(G) \backslash L(S)}\left(p^{m\left(\lambda_{l}+d\right)} \frac{\Psi\left(\lambda_{l}+d\right)}{\Psi(d)}\right),
$$

a $p^{-\operatorname{Ind}(S)} F_{S}(q ; \lambda ; m, M)$ - полином от $p^{\lambda_{l}}$. Частный случай произведения (4) есть $f_{G}\left(\lambda ; p^{-1}, 1\right) \prod_{l \in L(G)}\left(1-p^{\lambda_{l}+d}\right)$. Это замечание мы будем часто использовать в дальнейшем.

ЛЕмма 1. Пусть $G$ - произвольный связный граф. Для любъх параметров $m>0$ и $M<\infty$ произведения

$$
\begin{gathered}
g_{G}(\lambda ; M, \infty) \prod_{H \in \mathcal{H}}\left(1-p^{\operatorname{Ind}(H)}\right), \\
f_{G}(\lambda ; M, \infty) \prod_{l \in L(G)}\left(1-p^{\lambda_{l}+d}\right) \prod_{H \in \mathcal{H}_{0}}\left(1-p^{\operatorname{Ind}(H)}\right), \\
f_{G}(\lambda ; 0, m) \prod_{l \in L(G)}\left(1-p^{\lambda_{l}+d}\right) \prod_{Q \in \mathcal{Q}}\left(1-p^{\operatorname{Ind}(Q)}\right)
\end{gathered}
$$

- целье функиии от переменных $\lambda$. 
ДОКАЗАТЕЛЬСТВО ЛЕММЫ 1. Поскольку

$$
\begin{aligned}
g_{G}(\lambda ; M, \infty) & =M^{\operatorname{Ind}(G)} g_{G}(\lambda ; 1, \infty), \\
f_{G}(\lambda ; M, \infty) & =M^{\operatorname{Ind}(G)} f_{G}(\lambda ; 1, \infty), \\
f_{G}(\lambda ; 0, m) & =m^{\operatorname{Ind}(G)} f_{G}(\lambda ; 0,1),
\end{aligned}
$$

то без ограничения обшности можно считать, что $M=1, m=1$. Легко видеть, что если граф $G$ одночастично приводим, то $f_{G} \equiv g_{G} \equiv 0$. Если же граф $G$ распадается на блоки (компоненты двусвязности) $G_{1}, \ldots, G_{n}$, то

$$
g_{G}(\cdot)=\prod_{i=1}^{n} g_{G_{i}}(\cdot), \quad f_{G}(\cdot)=\prod_{i=1}^{n} f_{G_{i}}(\cdot)=\prod_{i=1}^{n} f_{G /\left(G \backslash G_{i}\right)}(\cdot),
$$

где $G \backslash G_{i}$ - дополнение графа $G_{i}$. Для вычисления интегралов, индексируемых каждым из графов $G_{i}$, можно воспользоваться рекуррентными формулами (см. формулу (4) и теоремы $2,2^{\prime}$ в [17])

$$
\begin{aligned}
g_{G}(\lambda ; 1, \infty) & =-\Psi(\operatorname{Ind}(G)) \sum_{\substack{H \in \mathcal{L}(G), H \neq G}} g_{G / H}\left(\lambda ; p^{-1}, 1\right) g_{H}(\lambda ; 1, \infty), \\
f_{G}(\lambda ; 1, \infty) & =-\Psi(\operatorname{Ind}(G)) \sum_{\substack{H \in P(G) \cap \mathcal{L}(G), H \neq G}} f_{G / H}\left(\lambda ; p^{-1}, 1\right) f_{H}(\lambda ; 1, \infty), \\
f_{G}(\lambda ; 0,1) & =\Psi(\operatorname{Ind}(G)) \sum_{\substack{H \in P(G) \cap \mathcal{L}(G), L(H) \neq \emptyset}} p^{-\operatorname{Ind}(G / H)} f_{G / H}(\lambda ; 0,1) f_{H}\left(\lambda ; p^{-1}, 1\right),
\end{aligned}
$$

где $\Psi(\alpha)=\left(1-p^{-\alpha}\right)^{-1}$.

Формулы (5)-(8) позволяют выразить интегралы $g_{G}(\lambda ; 1, \infty), f_{G}(\lambda ; 1, \infty), f_{G}(\lambda ; 0,1)$ в виде сумм произведений функций $\Psi, f_{G}\left(\lambda ; p^{-1}, 1\right)$ и функций, не зависяших от $\lambda$. Утверждение леммы 1 следует непосредственно из этих выражений.

Пусть $G$ - связный граф, имеюший одну внешнюю вершину $v_{0}$. Обозначим через $P_{0}(G)$ часть $P(G)$, состояшую из подграфов, имеющих в качестве одной из компонент связности подграф̆ с множеством вершин $\left\{v_{0}\right\}$.

Лемма 2. Пусть $G$ - произвольный связный граф, имеющий лишь одну внешнюю вериину, $0<m<\infty$ и

$$
\widehat{f}_{G}(\lambda ; m)=\sum_{H \in P_{0}(G)} f_{G / H}(\lambda ; 0, m) f_{H}(\lambda ; m, \infty) .
$$

Тогда произведение

$$
\widehat{f}_{G}(\lambda ; m) \prod_{l \in L(G)}\left(1-p^{\lambda_{l}+d}\right) \prod_{H \in \mathcal{H}_{0}}\left(1-p^{\operatorname{Ind}(H)}\right) \prod_{Q \in \mathcal{Q}_{0}^{\prime}}\left(1-p^{\operatorname{Ind}(Q)}\right)
$$

- целая функиия от переменных $\lambda$. 
ДокАЗАТЕЛЬСтво. Лемму 2 мы будем доказывать индукцией по $|V(G)|$. Воспользуемся леммой 1 работы [17], утверждаюшей, что для любого нетривиального графа $G$

$$
\sum_{H \in P(G)} f_{G / H}(\lambda ; 0, m) f_{H}(\lambda ; m, \infty)=0
$$

отсюда

$$
\begin{aligned}
\widehat{f}_{G}(\lambda ; m) & =-\sum_{H \in P(G) \backslash P_{0}(G)} f_{G / H}(\lambda ; 0, m) f_{H}(\lambda ; m, \infty)= \\
& =-\sum_{H^{\prime}} \widehat{f}_{G / H^{\prime}}(\lambda ; m) f_{H^{\prime}}(\lambda ; m, \infty),
\end{aligned}
$$

где суммирование в последнем выражении ведется по всем полным связным нетривиальным подграфам, содержашим внешнею вершину графра $G$. Утверждение леммы 2 следует из индукционного предположения и леммы 1 настояшей работы.

ДОКАЗАТЕЛЬСТВО ТЕОРЕМЫ 1 . Из формулы (2) и соотношения

$$
f_{G}(\lambda ; 0, M)=\sum_{H \in P(G)} f_{G / H}(\lambda ; 0, m) f_{H}(\lambda ; m, M)
$$

(формула (6) работы [17]) следует, что

$$
F_{G}(q ; \lambda)=\sum_{H_{1} \in \mathcal{H}_{0}^{\prime}} \sum_{H_{2} \in P\left(H_{1}\right)} \widehat{f}_{G / H_{1}}(\lambda ; m) \Phi_{H_{1} / H_{2}}(q ; \lambda ; m, M) f_{H_{2}}(\lambda ; M, \infty),
$$

где $\mathcal{H}_{0}^{\prime}$ - совокупность всевозможных подграфов $H$ таких, что $H \in P^{\prime}(G)$ и $\left|V\left(H^{\prime}\right)\right|=1$ для всех компонент связности $H^{\prime}$ граффа $H$, кроме компоненты с множеством вершин $V^{\prime} \supseteq$ $V_{\text {ext }}$. Утверждение теоремы 1 следует из последней формулы, лемм 1,2 и замечания перед ними.

Множитель $\prod_{l \in L(G)}\left(1-p^{\lambda_{l}+d}\right) \prod_{H \in \mathcal{H}_{0}}\left(1-p^{\mathrm{Ind}(H)}\right)$ в формуле $(3)$ связан с УФрасходимостями фейнмановской амплитуды, и его нули определяют УФ-серию полюсов интеграла $F_{G}(q ; \lambda)$. Аналогично значения $\lambda_{l}, l \in L(G)$, удовлетворяющие равенству $\operatorname{Ind}(Q)+2 \pi i n(\ln p)^{-1}=0$ для некоторых $Q \in \mathcal{Q}_{0}^{\prime}$ и $n \in Z$, определяют ИК-серию полюсов.

Утверждение, близкое по формулировке к теореме 1 , в вешественном случае было получено в работе Спира [12] (аналог функции $\Psi$ есть обычная Г-функция половинного аргумента).

Пусть $V_{\text {in }}(G)$ - множество всех внутренних вершин графа $G$.

СЛЕДСТВИЕ ТЕОРЕМЫ 1. Пусть $\lambda_{l}=\lambda_{l}^{*}+\varepsilon, \quad l \in L(G)$, әде $\lambda_{l}^{*}$ - вещественные числа, удовлетворяющие соотношениям

$$
\lambda_{l}^{*}>-d \text { для всех } l \in L(G), \quad \sum_{l \in S t(v)}\left(\lambda_{l}^{*}+d\right)=2 d \text { для всех } v \in V_{\mathrm{in}}(G) .
$$

Тогда $F_{G}(q ; \lambda)$ не имеет ИК-полюсов при $\varepsilon=0$.

ДокАЗАТЕЛЬСТво. Если $Q \in \mathcal{Q}_{0}^{\prime}$, то

$$
\left.\operatorname{Ind}(Q)\right|_{\lambda=\lambda^{*}}=\sum_{l \in L(Q)}\left(\lambda_{l}^{*}+d\right)-d(|V(Q)|-1)=
$$




$$
\begin{aligned}
& =\sum_{\substack{v \in V(Q), v \neq v_{0}}} \frac{\sum_{l \in S t(v)}\left(\lambda_{l}^{*}+d\right)}{2}-d(|V(Q)|-1)+\sum_{l \in L(Q)}\left|\imath_{l}^{v_{0}}\right|\left(\lambda_{l}^{*}+d\right) / 2= \\
& =\sum_{l \in L(Q)}\left|l_{l}^{v_{0}}\right|\left(\lambda_{l}^{*}+d\right) / 2>0,
\end{aligned}
$$

где $v_{0}$ - единственная внешняя вершина графа $Q,\left(l_{l}^{v}\right)$ - матрица инцидентности графа $G$.

Пусть теперь $\lambda^{*}=\left(\lambda_{l}^{*}, l \in L(G)\right)$ - полюс функции $g_{G}(\lambda ; m, \infty)$, или функции $f_{G}(\lambda ; m, \infty)$, или же функшии $f_{G}(\lambda ; 0, M)$. Пусть $\lambda_{l}=\lambda_{l}^{*}+\varepsilon, l \in L(G)$. Обозначим через $S$ оператор взятия главной части ряда Лорана мероморфной функции по переменной $\varepsilon$. Лемму 1 дополняет следуюшее утверждение, которое мы также будем использовать в дальнейшем

Лемма 3. Пусть $G-$ произвольный связный граф. Имеем

$$
S\left(\varepsilon^{\beta(G)} g_{G}(\lambda ; M, \infty)\right)=S\left(\varepsilon^{\beta(G)} g_{G}(\lambda ; 0, m)\right)=0 .
$$

Если к тому же $p^{\lambda_{l}^{*}+d} \neq 1$ для всех $l \in L(G)$, то

$$
S\left(\varepsilon^{|V(G)|-1} f_{G}(\lambda ; M, \infty)\right)=S\left(\varepsilon^{|V(G)|-1} f_{G}(\lambda ; 0, m)\right)=0 .
$$

ДокАЗАТЕЛЬСТво ЛЕммы 3 достаточно провести для случаев $m=1, \quad M=1$. Это доказательство легко получается индукцией по $|L(G)|$ для функции $g$ и индукцией по $|V(G)|$ для функции $f$, если воспользоваться формулами $(7),(8)$ для функции $f$, “УФ-формулой” (6) и аналогичной ей "ИК-формулой” для функции $g$. Последняя имеет вид:

$$
g_{G}(\lambda ; 0,1)=\Psi(\operatorname{Ind}(G)) \sum_{\substack{H \in \mathcal{L}(G), L(H) \neq \emptyset}} p^{-\operatorname{Ind}(G / H)} g_{G / H}(\lambda ; 0,1) g_{H}\left(\lambda ; p^{-1}, 1\right) .
$$

Рассмотрим, наконец, аналитические свойства $\Phi$ С с обрезанным пропагатором. Из леммы 1 и формул

$$
F_{G}(q ; \lambda ; m, \infty)=\sum_{H \in \mathcal{P}(G)} F_{G / H}(q ; \lambda ; m, R) g_{H}(\lambda ; R, \infty), \quad R \geq \max _{l \in E}\left\|q_{l}\right\|,
$$

(см. введение к работе [17]) и

$$
F_{G}(q ; \lambda ; 0, M)=\sum_{H \in P^{\prime}(G)} f_{G / H}(\lambda ; 0, r) \Phi_{H}(q ; \lambda ; r, M), \quad r<\min _{E^{\prime} \subset E}\left\|\sum_{l \in E^{\prime}} q_{l}\right\|,
$$

(см. теорему $1^{\prime}$ той же работы) следует теорема:

Теорема 2. Пусть $G$ - произвольный связный граф, $m>0, \quad M<\infty$. Тогда

$$
\begin{gathered}
F_{G}(q ; \lambda ; m, \infty) \prod_{H \in \mathcal{H}}\left(1-p^{\operatorname{Ind}(H)}\right), \\
F_{G}(q ; \lambda ; 0, M) \prod_{l \in L(G)}\left(1-p^{\lambda_{l}+d}\right) \prod_{Q \in \mathcal{Q}^{\prime}}\left(1-p^{\operatorname{Ind}(Q)}\right)
\end{gathered}
$$

- целье функции от переменных $\lambda$. 
Заметим, что полюсы сматривались в работе [13]. Аналитические свойства вешественных интегралов с пропагатором, обрезанным в нуле, аналогичны свойствам амплитуд с ненулевой массой.

\section{3. $R^{*}$-ОПЕРАЦИЯ}

Для более последовательного введения $R^{*}$-операции нам удобно сначала рассмотреть перенормировку ФИ с пропагатором, обрезанным в нуле или на бесконечности.

Пусть $\lambda^{*}$-полюс функции $F_{G}(q ; \lambda ; m, \infty)$ или же функции $F_{G}(q ; \lambda ; 0, M)$ и пусть $\lambda=$ $\lambda^{*}+\varepsilon$, где $\varepsilon-$ комплексное число. Сопоставим каждому связному подграфу $H$ графа $G$ некоторый полином

$$
O(H)=\sum_{\beta(H) \leq j \leq-1} a_{j} \varepsilon^{j},
$$

зависящий только от $H$ и $\lambda^{*}$. Аналогично каждому редуцированному графуу $Q=G / H$, $H \in \mathcal{P}^{\prime}(G)$, сопоставим полином

$$
\widetilde{O}(Q)=\sum_{\beta(Q) \leq j \leq-1} \tilde{a}_{j} \varepsilon^{j}
$$

При этом $O(H)=1(\widetilde{O}(Q)=1)$, если граф̆ $H$ (графф $Q)$ тривиален. Определим оператор аналитической перенормировки $R^{a r} \equiv R$ по формуле

$$
R F_{G}=\sum_{\pi \in \mathcal{P}(G)} F_{\left.G\right|_{\pi}} \prod_{H \in \pi} O(H) .
$$

Здесь $\pi$ есть набор компонент связности подграфа $H \in \mathcal{P}(G)$, а $\left.G\right|_{\pi}$ - редуцированный граф $G / H . \widetilde{R}$-операция определяется как

$$
\widetilde{R} F_{G}=\sum_{H \in \mathcal{P}^{\prime}(G)} \widetilde{O}(G / H) F_{H}
$$

В частности,

$$
\widetilde{R} g_{G}=\sum_{H \in \mathcal{P}(G)} \widetilde{O}(G / H) g_{H}
$$

Теорема 3. Пусть $G$ - произвольный связный граф, $m>0, \quad M<\infty$. Существует такой набор полиномов $O(H), \widetilde{O}(H)$, при котором интеграль $R F_{G}(q ; \lambda, m, \infty)$ и $\widetilde{R} F_{G}(q ; \lambda ; 0, M)$ являются мероморфными функииями переменной $\varepsilon$, аналитичными при $\varepsilon=0$. При әтом $O(H), \widetilde{O}(H)$ равны нулю, если граф одночастично приводим или $\operatorname{Re}\left(\left.\operatorname{Ind}(H)\right|_{\lambda_{2 *} *}\right) \neq 0$. Eсли граф $H$ распадается на блоки $H_{1}, \ldots, H_{n}$, то $O(H)=\prod_{i=1}^{n} O\left(H_{i}\right), \widetilde{O}(H)=\prod_{i=1}^{n} \widetilde{O}\left(H_{i}\right)$.

Рассмотрим всевозможные вакуумные $Ф И$ с обрезанным пропагатором $g_{H}(\lambda ; m, \infty)$, $H \in \mathcal{P}(G)$ и $g_{Q}(\lambda ; 0, M), Q=G / H, H \in \mathcal{P}^{\prime}(G)$. Заметим, что если $H^{\prime} \in \mathcal{P}(Q) \equiv$ $\left\{H^{\prime} \in \mathcal{P}(G): H^{\prime} \subseteq H\right\}$, то редуцированный граф $Q^{\prime}=Q / H^{\prime}$ совпадает с графом $G / H^{\prime}$. 
Лемма 4. Существует единственно возмохсный выбор полиномов $O(H)$, где $H \in \pi, \quad \pi \in \mathcal{P}(G)$, и полиномов $\widetilde{O}(Q)$, где $Q=G / H, H \in \mathcal{P}^{\prime}(G)$, при котором все вакуумные нормированные интеграль $R g_{H}(\lambda ; m, \infty)$, где $H \in \mathcal{P}(G)$, и $\widetilde{R} g_{Q}(\lambda ; 0, M)$, где $Q=G / H, \quad H \in \mathcal{P}^{\prime}(G)$, являются мероморфными функииями переменной $\varepsilon$, аналитичными при $\varepsilon=0$. При этом указанные полиномы обладают всеми свойствами, описанными в теореме 3 .

Заметим, что в случае $\varphi_{d}^{4}$-теории аналогичное утверждение для полиномов $O(H)$ было доказано в [5]. Мы рассмотрим доказательство леммы 4 и теоремы 3 только в ИКслучае. УФ-случай рассматривается аналогично.

ДОКАЗАТЕЛЬСТВо ЛЕмМЫ 4 (ИК-СЛУчАЙ). Заметим сначала, что единственно возможный выбор полиномов $\widetilde{O}(Q)$, при котором функции $\widetilde{R} g_{Q}(\lambda ; 0, M)$ будут аналитичны при $\varepsilon=0$, задается рекуррентным соотношением

$$
\widetilde{O}_{M}(Q)=-S \sum_{\substack{H^{\prime} \in \mathcal{P}(Q),\left|L\left(H^{\prime}\right)\right| \geq 1}} \widetilde{O}_{M}\left(Q / H^{\prime}\right) g_{H^{\prime}}(\lambda ; 0, M) .
$$

Здесь рекурсия ведется по мошности $L(Q)$; если $|L(Q)|=0$, то $\widetilde{O}(Q)=1$ по определению. Заметим, что равенство

$$
S\left(\varepsilon^{\beta(Q)} \widetilde{O}_{M}(Q)\right)=0
$$

следует из индукционного предположения и леммы 3 . Нам надо доказать, что полиномы $\widetilde{O}_{M}(Q)$ не зависят от $M$. Пусть операция $\widetilde{R}_{M}$ определяется по формуле (15), где в качестве $\widetilde{O}(Q)$ взяты полиномы $\widetilde{O}_{M}(Q)$. Воспользуемся формулой $(3)$ из работы $[17]$ :

$$
g_{H^{\prime}}(\lambda ; 0, M)=\sum_{H^{\prime \prime} \in \mathcal{P}\left(H^{\prime}\right)} g_{H^{\prime} / H^{\prime \prime}}(\lambda ; 0, m) g_{H^{\prime \prime}}(\lambda ; m, M),
$$

здесь $m<M$. Имеем

$$
\begin{aligned}
\widetilde{R}_{m} g_{Q}(\lambda ; 0, M) & =\sum_{H^{\prime} \in \mathcal{P}(Q)} \sum_{H^{\prime \prime} \in \mathcal{P}\left(H^{\prime}\right)} \widetilde{O}_{m}\left(Q / H^{\prime}\right) g_{H^{\prime} / H^{\prime \prime}}(\lambda ; 0, m) g_{H^{\prime \prime}}(\lambda ; m, M)= \\
& =\sum_{H^{\prime \prime} \in \mathcal{P}(Q)} g_{H^{\prime \prime}}(\lambda ; m, M) \sum_{H^{\prime} \in \mathcal{P}\left(Q / H^{\prime \prime}\right)} \widetilde{O}_{m}\left(Q / H^{\prime}\right) g_{H^{\prime} / H^{\prime \prime}}(\lambda ; 0, m)= \\
& =\sum_{H^{\prime \prime} \in \mathcal{P}(Q)} g_{H^{\prime \prime}}(\lambda ; m, M) \widetilde{R}_{m} g_{Q / H^{\prime \prime}}(\lambda ; 0, m) .
\end{aligned}
$$

Поскольку все функции в последней сумме аналитичны при $\varepsilon=0$, то $\widetilde{O}_{M}(Q) \equiv$ $\widetilde{O}_{m}(Q) \equiv \widetilde{O}(Q)$ для всех $Q=G / H, H \in \mathcal{P}^{\prime}(G)$.

Докажем теперь, что полиномы $\widetilde{O}(Q), \quad Q=G / H$ равны нулю, если $\operatorname{Re}\left(\left.\operatorname{Ind}(Q)\right|_{\varepsilon=0}\right) \neq 0$. Доказательство будем вести индукцией по $|L(Q)|$. Допустим, что это верно для всех граффов $Q^{\prime}=G / H^{\prime}, H^{\prime} \supset H$ и $\operatorname{Re}\left(\left.\operatorname{Ind}(Q)\right|_{\varepsilon=0}\right) \neq 0$. Имеем $\widetilde{O}(Q)=\widetilde{O}_{M}(Q)$, где

$$
O_{M}(Q)=-S \sum_{\substack{H^{\prime} \in \mathcal{P}(Q),\left|L\left(H^{\prime}\right)\right| \geq 1}} \widetilde{O}\left(Q / H^{\prime}\right) g_{H^{\prime}}(\lambda ; 0, M)=
$$

3 Теоретическая и математическая физика, т. 106, № 2, 1996 г. 


$$
=-\left.S \sum_{\substack{H^{\prime} \in \mathcal{P}(Q),\left|L\left(H^{\prime}\right)\right| \geq 1}} p^{M \operatorname{Ind}\left(H^{\prime}\right)}\right|_{\varepsilon=0} \widetilde{O}\left(Q / H^{\prime}\right) p^{M\left|L\left(H^{\prime}\right)\right| \varepsilon} g_{H^{\prime}}(\lambda ; 0,1) .
$$

В силу соотношения (17) вместо $p^{M\left|L\left(H^{\prime}\right)\right| \varepsilon}$ в последнем выражении можно подставить $\sum_{j=0}^{k}\left(M\left|L\left(H^{\prime}\right)\right| \varepsilon\right)^{j} / j !$, где $k \leq \beta(Q)$. Кроме того, вследствие предположения индукции для всех ненулевых слагаемых в последней сумме имеем

$$
\left\|p^{M \operatorname{Ind}\left(H^{\prime}\right)}\right\|_{\mid \varepsilon=0}=\left\|p^{M \operatorname{Ind}(Q)}\right\|_{\mid \varepsilon=0} .
$$

Устремив в равенстве (19) число $M$ к плюс- или к минус-бесконечности (к чему именно - зависит от знака $\left.-\operatorname{Re}\left(\left.\operatorname{Ind}(Q)\right|_{\varepsilon=0}\right)\right)$, получаем, что $\widetilde{O}(Q)=0$.

Пусть теперь граф $Q$ распадается на неприводимые подграфы $Q_{1}, \ldots, Q_{m}$. Формула

$$
\widetilde{O}(Q)=\prod_{i=1}^{m} \widetilde{O}\left(Q_{i}\right)
$$

легко доказывается индукцией по $|L(Q)|$, поскольку только при таком выборе $O(Q)$ имеет место равенство

$$
S\left(\widetilde{R} g_{Q}\right)=S\left(\prod_{i=1}^{m} \widetilde{R} g_{Q_{i}}\right)=0
$$

Если $|L(Q)|=1,|V(Q)|=2$, то равенство $\widetilde{O}(Q)=0$ очевидно. Из этого равенства и формулы $(20)$ следует, что $\widetilde{O}(Q)=0$ для любого одночастично приводимого графа $Q$. Лемма 4 доказана полностью.

ДОКАЗАТЕЛЬСТВО ТЕОРЕМЫ 3 (ИК-СЛУЧАЙ). Пусть $\widetilde{O}(Q)$ определяются по формуле (16). По определению

$$
\widetilde{R} F_{G}(\lambda ; 0, M)=\sum_{\pi \in \mathcal{P}^{\prime}(G)} \widetilde{O}\left(\left.G\right|_{\pi}\right) F_{\sigma(\pi)}(q ; \lambda ; 0, M) \prod_{\substack{H \in \pi, H \neq \sigma(\pi)}} g_{H}(\lambda ; 0, M),
$$

где через $\sigma(\pi)$ обозначен подграф $H$ из набора $\pi$, для которого $E(H)=E(G)$. Воспользуемся аналогом формулы (11) для ИК-случая:

$$
F_{\sigma(\pi)}(q ; \lambda ; 0, M)=\sum_{H^{\prime} \in \mathcal{P}^{\prime}(\sigma(\pi))} g_{\sigma(\pi) / H^{\prime}}(\lambda ; 0, m) F_{H^{\prime}}(q ; \lambda ; m, M),
$$

здесь $m<\min _{E^{\prime} \subset E(\sigma(\pi))}\left\|\sum_{l \in E^{\prime}} q_{l}\right\|$. Подставив $(22)$ и (18) в (21), получаем, что мероморфная функция

$$
\widetilde{R} F_{G}(\lambda ; 0, M)=\sum_{H^{\prime} \in \mathcal{P}^{\prime}(G)} F_{H^{\prime}}(q ; \lambda ; m, M) \widetilde{R} g_{G / H^{\prime}}(\lambda ; 0, m)
$$

аналитична по $\varepsilon$ при $\varepsilon=0$. Теорема доказана.

Заметим, что полиномы $O(H), H \in \pi, \pi \in \mathcal{P}(G)$, могут быть найдены с помощью рекуррентного соотношения

$$
O(H)=-S \sum_{\substack{\pi \in \mathcal{P}(H),|\pi|>1}} g_{\left.H\right|_{\pi}}(\lambda ; 1, \infty) \prod_{H^{\prime} \in \pi} O\left(H^{\prime}\right) .
$$


В следуюшем разделе мы дадим более эффективный алгоритм для нахождения вершинных частей в случае, если $p^{\lambda_{l}^{*}+d} \neq 1$ для всех $l \in L(G)$.

Пусть теперь $\lambda^{*}=\left(\lambda_{l}^{*}, l \in L(G)\right)$ - УФ- или (и) ИК-полюс функции $F_{G}(q ; \lambda)$ и пусть $\lambda=\lambda^{*}+\varepsilon \cdot R^{*}$-операция определяется как композиция $R$ - и $\widetilde{R}$-операций, определяемых по формулам (13) и (14):

$$
\begin{aligned}
R^{*} F_{G}(q ; \lambda) & =\widetilde{R} R F_{G}(q ; \lambda)= \\
& =\sum_{H^{\prime} \in \mathcal{P}^{\prime}(G)} \sum_{\pi \in \mathcal{P}\left(H^{\prime}\right)} \widetilde{O}\left(G / H^{\prime}\right) F_{\left.H^{\prime}\right|_{\pi}}(q ; \lambda ; 0, \infty) \prod_{H^{\prime \prime} \in \pi} O\left(H^{\prime \prime}\right) .
\end{aligned}
$$

Полиномы $O(H), H \in \pi, \pi \in \mathcal{P}(G)$, и $\widetilde{O}(G / H), H \in \mathcal{P}^{\prime}(G)$, определены выше.

ТЕОрема 4. Пусть $G$ - связный граф, не содержащий отделимых подграфов, и $|E(G)| \geq 2$. Тогда $R^{*}$-нормированный интеграл $R^{*} F_{G}(q ; \lambda)$ является мероморфной функиией $\varepsilon$, аналитичной при $\varepsilon=0$.

ДокаЗАтеЛьство. Подставим вместо каждого интеграла $F_{\left.H^{\prime}\right|_{\pi}}(q ; \lambda ; m, M)$ его представление (1) в виде линейной комбинации $Ф И$ с ограниченным пропагатором. Имеeм

$R^{*} F_{G}(q ; \lambda)=\sum_{H_{1} \in \mathcal{P}^{\prime}(G)} \widetilde{R} g_{G / H_{1}}(\lambda ; 0, m) \sum_{\pi \in \mathcal{P}\left(H_{1}\right)} F_{H_{1} \mid \pi}(q ; \lambda ; m, M) \prod_{H_{2} \in \pi} R g_{H_{2}}(\lambda ; M, \infty)$.

В силу леммы 4 последнее выражение аналитично по $\varepsilon$ в некоторой окрестности начала координат. Теорема доказана.

СЛЕДСТВИЕ ТЕОРЕМЫ 4. Пусть $G$ - связный граф, не содержсащий отделимых подграфов, $|E(G)| \geq 2$, и пусть $\lambda=\lambda^{*}+\varepsilon$, где $\lambda^{*}-$ вещественный вектор, удовлетворяющий соотношениям (9). Тогда $R$-нормированный интеграл $R F_{G}(q ; \lambda)$ является мероморфной функиией $\varepsilon$, аналитичной при $\varepsilon=0$.

ДоказАтельство. Докажем сначала, что $R$-нормированный $\Phi И$

$$
R F_{G}(q ; \lambda)=\sum_{\pi \in \mathcal{P}(G)} F_{\left.G\right|_{\pi}}(q ; \lambda) \prod_{H \in \pi} O(H)
$$

представляет собой линейную комбинацию интегралов $F_{\left.G\right|_{\pi}}(q ; \lambda)$, каждый из которых удовлетворяет условиям (9). Заметим, что ненулевые слагаемые в сумме (23) соответствуют наборам $\pi \in P(G)$, для которых $\left.\operatorname{Ind}(H)\right|_{\lambda=\lambda^{*}}=0$ для всех $H \in \pi$. Мы должны доказать, что если вершины полного связного графа $H$ являются внутренними вершинами графа $G$ и $\left.\operatorname{Ind}(H)\right|_{\lambda=\lambda^{*}}=0$, то

$$
\sum_{l \in L(G)} \sum_{v \in V(H)}\left|\imath_{l}^{v}\right|\left(\lambda_{l}^{*}+d\right)-\sum_{l \in L(H)}\left(\lambda_{l}^{*}+d\right)=2 d .
$$

Действительно,

$$
\sum_{l \in L(G)} \sum_{v \in V(H)}\left|\imath_{l}^{v}\right|\left(\lambda_{l}^{*}+d\right)-\sum_{l \in L(H)}\left(\lambda_{l}^{*}+d\right)=
$$




$$
\begin{aligned}
& =\sum_{v \in V(H)} \sum_{l \in S t(v)}\left(\lambda_{l}^{*}+d\right)-2 \sum_{l \in L(H)}\left(\lambda_{l}^{*}+d\right)= \\
& =2\left(d|V(H)|-d|L(H)|-\sum_{l \in L(H)} \lambda_{l}^{*}\right)=2\left(d-\left.\operatorname{Ind}(H)\right|_{\lambda=\lambda^{*}}\right)=2 d .
\end{aligned}
$$

Для доказательства утверждения следствия достаточно теперь доказать равенство $\widetilde{R} F_{G}(q ; \lambda)=F_{G}(q ; \lambda)$. Оно вытекает из отсутствия ИК-полюсов у интеграла $F_{G}(q ; \lambda)$.

\section{4. КОНТРЧЛЕНЫ В КООР ДИНАТНОМ И ИМПУЛЬСНОМ ПРОСТРАНСТВАХ}

Пусть $\Delta_{\lambda_{l}}(x)$ есть преобразование Фурье от функции $\left\|k_{l}\right\|^{\lambda_{l}}$. Оно имеет вид $\frac{\Psi\left(\lambda_{l}+d\right)}{\Psi\left(-\lambda_{l}\right)}\|x\|^{-\lambda_{l}-d}, x \in Q_{p}^{d}$. Обозначим через $\widetilde{F}_{G}\left(x_{1}, \ldots, x_{|E|} ; \lambda ; m, M\right)$ интеграл

$$
\begin{aligned}
\int \prod_{l \in L(G)} \Delta_{\lambda_{l}}\left(x_{i(l)}-x_{f(l)}\right) \times & \\
& \times\left(\chi_{1 / m}\left(x_{i(l)}-x_{f(l)}\right)-\chi_{1 / M}\left(x_{i(l)}-x_{f(l)}\right)\right) \prod_{v \in V_{\text {in }}(G)} d x_{v}
\end{aligned}
$$

где $i(l)$ и $f(l)$ - соответственно начальная и конечная вершины ребра $l$. В дальнейшем для набора внешних переменных $\left(x_{1}, \ldots, x_{|E|}\right)$ мы будем использовать обозначение $y=$ $\left(y_{v}, v \in V_{\text {ext }}(G)\right)$, где $V_{\text {ext }}(G)=V(G)_{\sim} \backslash V_{\text {in }}(G)$. Как показано в [16, 17], аналитическое продолжение мероморфной функции $\widetilde{F}_{G}(y ; \lambda) \equiv \widetilde{F}_{G}(y ; \lambda ; 0, \infty)$ определено для любых значений параметров $\lambda$, если граф $G$ имеет не менее двух внешних вершин, не содержит отделимых подграфов и если

$$
y \notin \bigcup_{v \neq v^{\prime}}\left\{y \in Q_{p}^{d|E|}: y_{v}=y_{v^{\prime}}\right\} .
$$

Последнее условие мы будем предполагать выполненным во всех формулах ниже. Если $(m, M) \neq(0, \infty)$, то интеграл $\widetilde{F}_{G}(y ; \lambda ; m, M)$ определен для любого связного графа $G$, не содержашего лепестков. Если $\left|V_{\text {ext }}(G)\right|=1$, то этот интеграл является функцией от $\lambda$, но не от $y$. Эту функцию мы будем обозначать через $\tilde{f}_{G}(\lambda ; m, M)$. Заметим, что в работе [17] мы употребляли такое же обозначение для аналогичного интеграла с "пропагатором" $\|x\|^{-\lambda_{l}-d}$.

Пусть $\widetilde{P}(G)=\left\{\pi \in P(G):\left|V_{\text {ext }}(G) \cap V(H)\right| \leq 1\right.$ для всех $\left.H \in \pi\right\}$. Для $\pi \in \widetilde{P}(G)$ определим $\widetilde{\Phi}_{\left.G\right|_{\pi}}(y ; \lambda ; m, M)$ по формуле $(24)$, заменив в ней $G$ на $\left.G\right|_{\pi}$ и отождествив вершины, принадлежашие $V(H)$ для каждого $H \in \pi$. Положим, что $\tilde{f}_{\pi}(\lambda ; m, M)=$ $\prod_{H \in \pi} \tilde{f}_{H}(\lambda ; m, M)$. Формулы (11), (12) имеют следующие координатные аналоги (см. теоремы 3 и $3^{\prime}$ в [17]):

$$
\begin{aligned}
\widetilde{F}_{G}(y ; \lambda ; m, \infty) & =\sum_{\pi \in \widetilde{P}(G)} \widetilde{\Phi}_{\left.G\right|_{\pi}}(y ; \lambda ; m, R) \tilde{f}_{\pi}(\lambda ; R, \infty), \\
\widetilde{F}_{G}(y ; \lambda ; 0, M) & =\sum_{\pi \in P^{\prime}(G)} \tilde{f}_{\left.G\right|_{\pi}}(\lambda ; 0, r) \widetilde{F}_{\sigma(\pi)}(y ; \lambda ; r, M) \prod_{H \in \pi \backslash\{\sigma(\pi)\}} \tilde{f}_{H}(\lambda ; r, M),
\end{aligned}
$$


здесь $R>\left(\min _{v \neq v^{\prime}}\left\|y_{v}-y_{v^{\prime}}\right\|\right)^{-1}, r \leq\left(\max _{v \neq v^{\prime}}\left\|y_{v}-y_{v^{\prime}}\right\|\right)^{-1}$.

Обозначим через $C_{G}\left(p^{d}\right)$ число способов раскраски вершин графа $G$ с помощью $p^{d}$ красок таким образом, чтобы смежные вершины имели разные цвета (хроматический многочлен графа $G[18])$. Пусть $C_{G}^{\prime}=\gamma(G) C_{G}\left(p^{d}\right) / p^{d|V(G)|}$, где $\gamma(G)=$ $\prod_{l \in L(G)} \frac{\Psi\left(\lambda_{l}+d\right)}{\Psi\left(-\lambda_{l}\right)}$. Вакуумные интегралы могут быть найдены с помошью следуюших рекуррентных соотношений (теоремы 4 и $4^{\prime}$ в [17]):

$$
\begin{aligned}
\tilde{f}_{G}(\lambda ; 1, \infty) & =\Psi(-\operatorname{Ind}(G)) \sum_{\substack{\pi \in P(G),|\pi|>1}} p^{\operatorname{Ind}(G)-\operatorname{Ind}\left(\left.G\right|_{\pi}\right)} C_{\left.G\right|_{\pi}}^{\prime} \tilde{f}_{\pi}(\lambda ; 1, \infty), \\
\tilde{f}_{G}(\lambda ; 0,1) & =-\Psi(-\operatorname{Ind}(G)) \sum_{\substack{\pi \in P(G),|\pi|<|V(G)|}} \tilde{f}_{\left.G\right|_{\pi}}(\lambda, 0,1) \prod_{H \in \pi} C_{H}^{\prime} .
\end{aligned}
$$

Эти формулы могут быть преобразованы. Пусть $V^{\prime} \subseteq V(G)$. Обозначим через $H\left(V^{\prime}\right)$ полный подграфо графа $G$, натянутый на множество вершин $V^{\prime}$. Имеет место рекуррентное соотношение (см. $[6,5,7,16])$

$$
\begin{aligned}
\tilde{f}_{G}(\lambda ; 1, \infty)=\Psi( & -\operatorname{Ind}(G)) \sum_{\substack{V(G)=\cup_{i=1}^{n} V_{i}, n>1}} c(n) \gamma\left(\left.G\right|_{\left\{H\left(V_{1}\right), \ldots, H\left(V_{n}\right)\right\}}\right) \times \\
& \times \prod_{i} p^{\operatorname{Ind}\left(H\left(V_{i}\right)\right)} \tilde{f}_{H\left(V_{i}\right)}(\lambda ; 1, \infty),
\end{aligned}
$$

где $c(n)=\left(1-p^{-d}\right)\left(1-2 p^{-d}\right) \ldots\left(1-(n-1) p^{-d}\right)$ и суммирование ведется по всем разбиениям множества $V(G)$ на непересекающиеся подмножества $V_{1}, \ldots, V_{n}, n>1$. Подобная формула в ИК-случае имеет вид

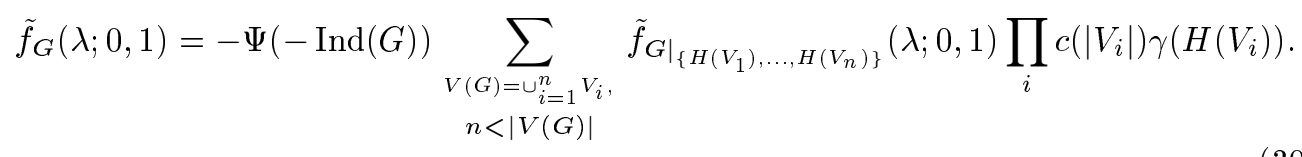

Она следует из формулы (28) и из предложения 2 работы [17].

Используя формулы $(25),(26),(27),(28)$, легко доказать координатные аналоги теорем 1 и 2.

ТЕОрема 5. Пусть $G-$ связный граф, не содержащий отделимых подграфов, $\left|V_{\text {ext }}(G)\right| \geq 2$, и пусть $\widetilde{\mathcal{H}}_{0}=\mathcal{H}_{0} \cap\left\{H \subseteq G:\left|V_{\text {ext }}(G) \cap V(H)\right| \leq 1\right\}$. Тогда

$$
\widetilde{F}_{G}(y ; \lambda) \prod_{l \in L(G)}\left(1-p^{\lambda_{l}+d}\right) \prod_{H \in \widetilde{\mathcal{H}}_{0}}\left(1-p^{\operatorname{Ind}(H)}\right) \prod_{Q \in \mathcal{Q}_{0}^{\prime}}\left(1-p^{\operatorname{Ind}(Q)}\right)
$$

- целая функиия от переменных $\lambda$.

ТЕОРема 6. Пусть $G$ - связньй граф, не содержсащий лепестков, $m>0$, $M<\infty$. Тогда

$$
\begin{aligned}
& F_{G}(q ; \lambda ; m, \infty) \prod_{l \in L(G)}\left(1-p^{\lambda_{l}+d}\right) \prod_{H \in \widetilde{\mathcal{H}}_{0}}\left(1-p^{\operatorname{Ind}(H)}\right), \\
& F_{G}(q ; \lambda, 0, M) \prod_{l \in L(G)}\left(1-p^{\lambda_{l}+d}\right) \prod_{Q \in \mathcal{Q}^{\prime}}\left(1-p^{\operatorname{Ind}(Q)}\right)
\end{aligned}
$$


- целье функиии от переменных $\lambda$.

Пусть

$$
p^{\lambda_{l}^{*}+d} \neq 1 \text { для всех } l \in L(G)
$$

и пусть $\lambda=\lambda^{*}+\varepsilon$. Условие (31) мы будем предполагать выполенным во всей оставшейся части статьи. Оно необходимо для конечности множителя $\gamma(G)$.

Определим полиномы $Y(H)$ для всех связных подграфов $H$ граффа $G$ с помошью рекуррентной формулы

$$
Y(H)=-S \sum_{\substack{\pi \in P(H),|\pi|>1}} \tilde{f}_{\left.H\right|_{\pi}}(\lambda ; 1, \infty) \prod_{H^{\prime} \in \pi} Y\left(H^{\prime}\right)
$$

Каждому редуцированному графу $Q=G / H, H \in \mathcal{P}^{\prime}(G)$, сопоставим полином

$$
\tilde{Y}(Q)=-S \sum_{\substack{\pi \in P(Q),|\pi|<|V(Q)|}} \tilde{Y}\left(\left.Q\right|_{\pi}\right) \prod_{H^{\prime} \in \pi} \tilde{f}_{H^{\prime}}(\lambda ; 0,1) .
$$

Заметим, что $\tilde{Y}(G / H)=0$, если $H \in \mathcal{P}^{\prime}(G) \backslash P^{\prime}(G)$.

Определим операцию $R$ в координатном пространстве по формуле

$$
R \widetilde{F}_{G}=\sum_{\pi \in \widetilde{P}(G)} \widetilde{F}_{\left.G\right|_{\pi}} \prod_{H \in \pi} Y(H) .
$$

$\widetilde{R}$-операция в координатном пространстве определяется как

$$
\widetilde{R} \widetilde{F}_{G}=\sum_{\pi \in P^{\prime}(G)} \tilde{Y}\left(\left.G\right|_{\pi}\right) \prod_{H \in \pi} \widetilde{F}_{H} .
$$

Положим, что $R^{*} \widetilde{F}_{G}(y ; \lambda)=\widetilde{R} R \widetilde{F}_{G}(y ; \lambda)$, где операторы $R$ и $\widetilde{R}$ определяются по формулам (34) и (35).

ТЕОРема 7. Пусть $G$ - связный граф, выполнено условие (31) и $m>0, M<\infty$. Перенормированные интегралы $R \widetilde{F}_{G}(y ; \lambda, m, \infty), \widetilde{R} \widetilde{F}_{G}(y ; \lambda, 0, M)$ и $R^{*} \widetilde{F}_{G}(y ; \lambda)$ являются мероморфными функииями $\varepsilon$, аналитичными при $\varepsilon=0$. Полиномы $Y(H)$, $\tilde{Y}(H)$ имеют степень не более чем $|V(H)|-1$. Они равны нулю, если граф Н одночастично приводим или $\operatorname{Re}\left(\left.\operatorname{Ind}(H)\right|_{\lambda=\lambda^{*}}\right) \neq 0$. Eсли граф H распадается на блоки $H_{1}, \ldots, H_{n}, \operatorname{mo} Y(H)=\prod_{i=1}^{n} Y\left(H_{i}\right), \stackrel{\widetilde{Y}}{ }(H)=\prod_{i=1}^{n} \tilde{Y}\left(H_{i}\right)$.

ДоКАЗАТЕЛЬСТВо ТЕОРЕМЫ 7 полностью аналогично доказательству теорем 3,4 . Заметим, что утверждение о степени полиномов $Y$ и $\widetilde{Y}$ вытекает также из леммы 3 и следующей ниже теоремы 8 , являюшейся основным результатом данного раздела.

ТЕОрема 8. Пусть $G$ - связный граф и выполнено условие (31). Тогда имеет место тождественное равенство полиномов $O$ и $Y$, а также полиномов $\widetilde{O}$ и $\widetilde{Y}$. При этом выполняются рекуррентные соотношения

$$
O(H)=-S \sum_{\substack{\pi \in P(H) \\|\pi|>1}} f_{\left.H\right|_{\pi}}(\lambda ; 1, \infty) \prod_{H^{\prime} \in \pi} O\left(H^{\prime}\right)
$$


для всех связных подграфов $H$ графа $G$ и

$$
\widetilde{O}(G / H)=-S \sum_{\substack{H^{\prime} \in P(G), H^{\prime} \subset H}} \widetilde{O}\left(G / H^{\prime}\right) f_{H^{\prime}}(\lambda ; 0,1)
$$

для всех $H \in \mathcal{P}^{\prime}(G)$.

ДоказАТЕЛЬСтво. Если $p^{\lambda_{l}^{*}+d} \neq 1$, то для графа $Q$, состоящего из одного лепестка $l$, имеет место равенство $\widetilde{O}(Q)=0$. Из этого равенства и формулы $(20)$ следует, что

$$
\widetilde{O}(G / H)=0, \text { если } H \in \mathcal{P}^{\prime}(G) \backslash P^{\prime}(G) .
$$

Очевидно, что

$$
g_{H^{\prime}}(\lambda ; 0,1)=f_{H^{\prime}}(\lambda ; 0,1) .
$$

Подставив равенства (38) и (39) в (16) получим (37).

Обозначим через $M(G)$ множество всевозможных фактор-графов подграфов графа $G$. Пусть $h_{H}(\varepsilon), H \in M(G),-$ произвольные мероморфные по $\varepsilon$ функции, принимающее нулевое значение в случае наличия лепестков у графа $H$. Пусть $Z\left(H^{\prime}\right), H^{\prime} \in \pi$, $\pi \in \mathcal{P}(G),-$ некоторые полиномы от переменной $\varepsilon^{-1}$, причем $Z\left(H^{\prime}\right)=1$, если граф $H^{\prime}$ тривиален; в противном случае свободный член полинома $Z\left(H^{\prime}\right)$ равен нулю. Определим оператор $R_{Z}$, действуюший на функции $h_{H}, H \in \mathcal{P}(G)$, по формуле

$$
R_{Z} h_{H}=\sum_{\pi \in P(H)} h_{\left.H\right|_{\pi}} \prod_{H^{\prime} \in \pi} Z\left(H^{\prime}\right) \equiv \sum_{\pi \in \mathcal{P}(H)} h_{\left.H\right|_{\pi}} \prod_{H^{\prime} \in \pi} Z\left(H^{\prime}\right) .
$$

Легко видеть, что сушествует единственный выбор $Z_{h}\left(H^{\prime}\right)$ полиномов $Z\left(H^{\prime}\right)$, при котором все функции $R_{Z} h_{H}, \quad H \in \mathcal{P}(G)$, аналитичны в окрестности $\varepsilon=0$. Например, для функций $R f_{H}(\lambda ; 1, \infty)$ такой выбор задается формулой $(36)$, а для функций $R \tilde{f}_{H}(\lambda ; 1, \infty)$ - формулой (32).

Аналогичное утверждение верно и для $\widetilde{R}$-операции. Пусть $\widetilde{Z}(G / H), H \in P^{\prime}(G)$ некоторые полиномы от $\varepsilon^{-1}$, причем $\widetilde{Z}=1$, если $H=G$; в противном случае свободньй член полинома $\widetilde{Z}$ равен нулю. Определим оператор $\widetilde{R}_{\widetilde{Z}}$, действуюший на функции $h_{G / H}, H \in P^{\prime}(G)$, по формуле

$$
\widetilde{R}_{\widetilde{Z}} h_{Q}=\sum_{\pi \in P(Q)} \widetilde{Z}\left(\left.Q\right|_{\pi}\right) \prod_{H^{\prime} \in \pi} h_{H^{\prime}}
$$

Легко видеть, что сушествует единственный выбор полиномов $\widetilde{Z}$, при котором все функции $\widetilde{R}_{\widetilde{Z}} h_{G / H}, H \in P^{\prime}(G)$, аналитичны в окрестности $\varepsilon=0$.

Воспользуемся теперь теоремой П. 2 работы [17], утверждающей, что

$$
f_{G}(\lambda ; 0,1)=\sum_{H \in P(G)} \tilde{f}_{G / H}(\lambda ; 0,1) \prod_{l \in L(H)} \frac{\Psi\left(\lambda_{l}+d\right)}{\Psi(d)} .
$$

Отсюда следует, что функция

$$
\widetilde{R}_{\widetilde{Y}} f_{G / H}(\lambda ; 0,1)=\sum_{H^{\prime} \in P(G / H)}\left(R_{\widetilde{Y}} \tilde{f}_{G / H^{\prime}}(\lambda ; 0,1)\right) \prod_{l \in L\left(H^{\prime}\right)} \frac{\psi\left(\lambda_{l}+d\right)}{\Psi(d)}
$$


аналитична в окрестности $\varepsilon=0$ для всех $H \in P^{\prime}(G)$. Равенство полиномов $\widetilde{O}$ и $\tilde{Y}$ доказано.

В УФ-случае мы воспользуемся теоремой П. 1 работы [17], утверждающей, что

$$
\tilde{f}_{H}(\lambda ; 1, \infty)=\sum_{\pi \in P(H)} \prod_{l \in L\left(\left.H\right|_{\pi}\right)} \frac{\psi\left(\lambda_{l}+d\right)}{\Psi(d)} \prod_{H^{\prime} \in \pi} f_{H^{\prime}}(\lambda ; 1, \infty) .
$$

Используя соотношение

$$
f_{H^{\prime}}(\lambda ; 1, \infty)=\sum_{S \in \tau(G)} g_{S}(\lambda ; 1, \infty) \prod_{l \in L\left(H^{\prime}\right) \backslash L(S)}\left(\frac{\Psi\left(\lambda_{l}+d\right)}{\Psi(d)}\right),
$$

равенство (40) можно переписать в виде

$$
\tilde{f}_{H}(\lambda ; 1, \infty)=\sum_{H^{\prime} \in \mathcal{P}(H)} g_{H^{\prime}}(\lambda ; 1, \infty) \prod_{l \in L\left(H / H^{\prime}\right)} \frac{\Psi\left(\lambda_{l}+d\right)}{\Psi(d)} .
$$

Из равенства (40) следует, что функция

$$
R_{Z_{f}} \tilde{f}_{H}(\lambda ; 1, \infty)=\sum_{\pi \in P(H)} \prod_{l \in L\left(\left.H\right|_{\pi}\right)} \frac{\psi\left(\lambda_{l}+d\right)}{\Psi(d)} \prod_{H^{\prime} \in \pi}\left(R_{Z_{f}} f_{H^{\prime}}(\lambda ; 1, \infty)\right)
$$

аналитична в окрестности $\varepsilon=0$. С другой стороны,

$$
R_{O} \tilde{f}_{H}(\lambda ; 1, \infty)=\sum_{\pi \in \mathcal{P}(H)} \prod_{l \in L\left(\left.H\right|_{\pi}\right)} \frac{\psi\left(\lambda_{l}+d\right)}{\Psi(d)} \prod_{H^{\prime} \in \pi}\left(R g_{H^{\prime}}(\lambda ; 1, \infty)\right)
$$

Следовательно, $O\left(H^{\prime}\right)=Y\left(H^{\prime}\right)=Z_{f}\left(H^{\prime}\right)$ для всех связных подграффов $H^{\prime}$ граффа $G$, что и требовалось доказать.

Заметим, что теорема 8 и “УФ-формулы" (29), (32), “ИК-формулы" (30), (33) дают простой путь для вычисления контрчленов в импульсном пространстве.

Автор признателен М.Д. Миссарову за замечания, способствующие улучшению стиля изложения в данном цикле работ, а также В.А. Смирнову за замечания, благодаря которым удалось усилить результат теоремы 1.

Работа выполнена при поддержке Российского фонда фундаментальных исследований (проект 95-01-00270) и Международного научного фонда (код гранта RHA300).

\section{Список литературы}

[1] Владимиров В.С., Волович И.В., Зеленов Е.И. Р-адический анализ и математическая физика. М.: Наука, 1994.

[2] Brekke L., Freund P.G.O. // Phys. Rep. 1993. P. 2-66.

[3] Боголюбов Н.Н., Ширков Д.В. Введение в теорию квантованных полей. М.: Наука, 1984.

[4] Завьялов О.И. Перенормированные диаграммы Фейнмана. М.: Наука, 1979.

[5] Missarov M.D. Dynamical systems and statistical mechanics, ed. Ya.G. Sinai (Adv. Sov. Math. Vol. 3, Amer. Matn. Soc., 1991). P. 143-161.

[6] Lerner E.Yu., Missarov M.D. // Commun. Math. Phys. 1989. V. 121. P. 35-48.

[7] Smirnov V.A.// Commun. Math. Phys. 1992. V. 149. P. 623-636. 
[8] Смирнов B.A. Перенормировка и асимптотические разложения фейнмановских амплитуд. М.: Изд-во МГУ, 1990.

[9] Smirnov V.A.// Mod. Phys. Lett. A. 1991. V. 6. P. 1421-1427.

[10] Missarov M.D. // Lett. in Math. Phys. 1993. V. 27. P. 149-154.

[11] Missarov M.D.// Lett. in Math. Phys. 1994. V. 32. P. 347-356.

[12] Speer E.R. // Ann. Inst. H. Poincare. 1973. V. 23. P. 1-21.

[13] Блехер П.М. Препринт ИПМ АН СССР № 117. М.: 1979 (Rep. Math. Phys. 1984. V. 19. P. 117.)

[14] Смирнов В.А. // ТМФ. 1980. Т. 44. № 3. С. 307-320; 1981. Т. 46. № 2. С. 199-212.

[15] Аникин С.А., Завьялов О.И., Карчев Н.И. // ТМФ. 1980. Т. 44. № 3. С. 291-306; 1981. T. 46. № 1 . C. 3-26.

[16] Лернер Э.Ю. // ТМФ. 1995. Т. 102. № 3. С. 367-377.

[17] Лернер Э.Ю. // ТМФ. 1995. Т. 104. № 3. С. 371-392.

[18] Татm У.Т. Теория графов. М.: Мир, 1988.

[19] Смирнов В.А., Четыркин К.Г. Препринт НИИЯФ МГУ 89-2/79. М., 1989.

Казанский государственный

Поступила в редакцию университет

\section{E. Yu. Lerner}

FEYNMAN INTEGRALS OF $p$-ADIC ARGUMENT IN MOMENTUM SPACE. III. RENORMALIZATION

The ultraviolet and infrared sequences of the poles for the massless Feynman integrals of $p$-adic argument with the arbitrary power of propagators are found. Within the framework of MS-scheme for the analytic $R^{*}$-operation it is proved that the renormalized amplitudes are finite. Simple formulas for the calculation of counterterms (vertex parts) in coordinate and momentum spaces are given. 